\title{
Lectin-Positive Spherical Deposits (SPD) Detected in the Molecular Layer of Hippocampal Dentate Gurus of Schizophrenia
}

${\text { Keiko } \text { Ikemoto }^{1 *} \text { and Akiyoshi Nishimura }}^{2}$

${ }^{1}$ Department of Psychiatry, Iwaki Kyoritsu General Hospital, Iwaki, 973-8555, Japan

${ }^{2}$ Department of Forensic Medicine, Health Bioscience Institute, University of Tokushima Graduate School, Tokushima, 770-8503, Japan

\begin{abstract}
Lectins are proteins which specifically bind (or crosslink) carbohydrates. Recently, importance of glycosylation has been emphasized in pathogenesis of diseases. Lectin-positive spherical deposits (SPD), 3-10 micron in diameter, have been detected in the molecular layer of dentate gyrus of hippocampus in schizophrenia, dementia, Down's syndrome, and aged individuals. In schizophrenia, SPD was observed without exception, regardless of having history of pharmacotherapy. By using multi-labeling histochemical methods, single strand DNA was co-localized in hippocampal SPD of schizophrenia with lectin, including GSI-B4 for galactose, and UEA-I for fucose, suggesting that SPD formation in schizophrenia is related to apoptotic process. The molecular basis of SPD formation should further be investigated in brains with neuropsychiatric illnesses.
\end{abstract}

Keywords: Sugar chain; Postmortem brain; Apoptosis; Dentate gyrus; Hippocampus; Schizophrenia

\section{Introduction}

Hippocampal neuropathology, as a result of pathognomonic procedures of molecular basis related to memory and cognitive disturbance, has been described in mental disorders such as schizophrenia [1]. Glycoconjugates have been implicated to play a major role in the process of cell-cell recognition of development during embryogenesis. Recently, increasing number of diseases come to be known to be caused by abnormalities in the biosynthesis of sugar chains [2]. Abnormal accumulation or deposition of the sugar chains in brains of patients with neurodegenerative diseases has been reported [3].

Lectin staining is able to reveal several kinds of carbohydraterelated depositions in addition to the conventional degenerative changes including senile plaques, neurofibrillary tangles and corpora amylacea (Table 1, Figure 1) [4]. According to an old definition," Lectins are multivalent carbohydrate-binding proteins or glycoproteins except for enzymes and antibodies." As a significant number of exceptions are evident now, such a narrow definition, however, seems no longer relevant. To date, "lectins are defined as proteins which specifically bind (or crosslink) carbohydrates." The authors detected lectin-positive spherical deposits (SPD) in the molecular layer of hippocampal dentate gyrus of schizophrenia (Figures 1-3), dementia, Down's syndrome, and

\begin{tabular}{|c|c|c|}
\hline Lectin and antibodies & Abbreviation & $\begin{array}{l}\text { Carbohydrate binding } \\
\text { specificity }\end{array}$ \\
\hline Archis hypogaea agglutinin & PNA & $\operatorname{Gal}(\beta 1,3)$ GalNAc \\
\hline Canavalia ensiformis agglutinin & Con A & $\begin{array}{l}\text { Branched a-Man } \\
\text { ( Mana1-6 (Mana1-3) Man) }\end{array}$ \\
\hline Datura stramonium & DSA & GlcNAc \\
\hline Dolichos biflorus agglutinin & DBA & a-GalNAc \\
\hline Erythrina cristagalli & ECA & $\operatorname{Gal}(\beta 1,3) \mathrm{GlcNAc}$ \\
\hline Glycine max agglutinin & SBA & GalNAc( $\alpha 1,3)$ Gal \\
\hline Griffonia simplicifolia iso agglutinin I-B4 & GSI-B4 & a-Gal \\
\hline Pisum sativum agglutinin & PSA & Fuc( $\alpha 1,6) G l c, \alpha-M a n$ \\
\hline Triticum vulgare & WGA & $\begin{array}{l}\operatorname{Man} \beta(1,4) \operatorname{GlcNAc}(1,4) \\
\operatorname{GlcNAc}\end{array}$ \\
\hline Ulex europaeus agglutinin & UEA-I & a-Fuc \\
\hline
\end{tabular}

Table 1: Interaction between lectins and antibodies, abbreviation, and the carbohydrate binding specificity is shown. aged individuals (Figure 4) [4]. In the present mini-review, significance of lectin-positive SPD would be discussed.

\section{Materials and Methods}

Brain tissue sections from hippocampus were obtained at autopsy for pathological diagnosis in compliance with the ethical code of the Ethical Committee of the Japanese Society of Legal Medicine. In the present study, hippocampal sections from 51 individuals were examined, i.e., 16 cases of clinically diagnosed or under treatment of schizophrenia, 2 Down's syndrome, 6 dementia of Alzheimer' type, 5 tangle's dementia, and 22 individuals without having clinical history of neuropsychiatric diseases.

Procedures of preparation of human brain materials and immunohistochemistry have been described elsewhere [5]. The hippocampal sections were made by using a microslicer (DTK-3000, Dosaka EM) in 40 micron thick in coronal planes.

In multi-fluorescent staining, antibodies against glial fibrillary acidic protein (GFAP), CD45, neuro-filament (NF), and single strand DNA (ssDNA) and SYBR Green were used. DBA, GSI-B4, and UEA-I were used as lectins (Table 1). Details of procedures were described elsewhere [6].

In immuno-electron microscopical method, after embedding in Luveak-812, 60nm ultrathin sections were stained with uranyl acetate and lead citrate sequentially, and examined using Hitachi H-600 electron microscope. A floating method of lectin histochemical staining before embedding in Luveak-812 was using the same three lectins as multifluorescent staining. Details of procedures were described elsewhere [6].

*Corresponding author: Keiko Ikemoto, Department of Psychiatry, Iwaki Kyoritsu General Hospital, Iwaki, 973-8555, Japan, Tel: +81-246-26-3151; Fax: +81-246-272148; E-mail: ikemoto@iwaki-kyoritsu.iwaki.fukushima.jp

Received December 08, 2014; Accepted November 23, 2015; Published November 30, 2015

Citation: Ikemoto K, Nishimura A (2015) Lectin-Positive Spherical Deposits (SPD) Detected in the Molecular Layer of Hippocampal Dentate Gurus of Schizophrenia. J Neurol Neurophysiol 6: 328. doi:10.4172/2155-9562.1000328

Copyright: $\odot 2015$ lkemoto $\mathrm{K}$, et al. This is an open-access article distributed under the terms of the Creative Commons Attribution License, which permits unrestricted use, distribution, and reproduction in any medium, provided the original author and source are credited. 
Citation: Ikemoto K, Nishimura A (2015) Lectin-Positive Spherical Deposits (SPD) Detected in the Molecular Layer of Hippocampal Dentate Gurus of Schizophrenia. J Neurol Neurophysiol 6: 328. doi:10.4172/2155-9562.1000328

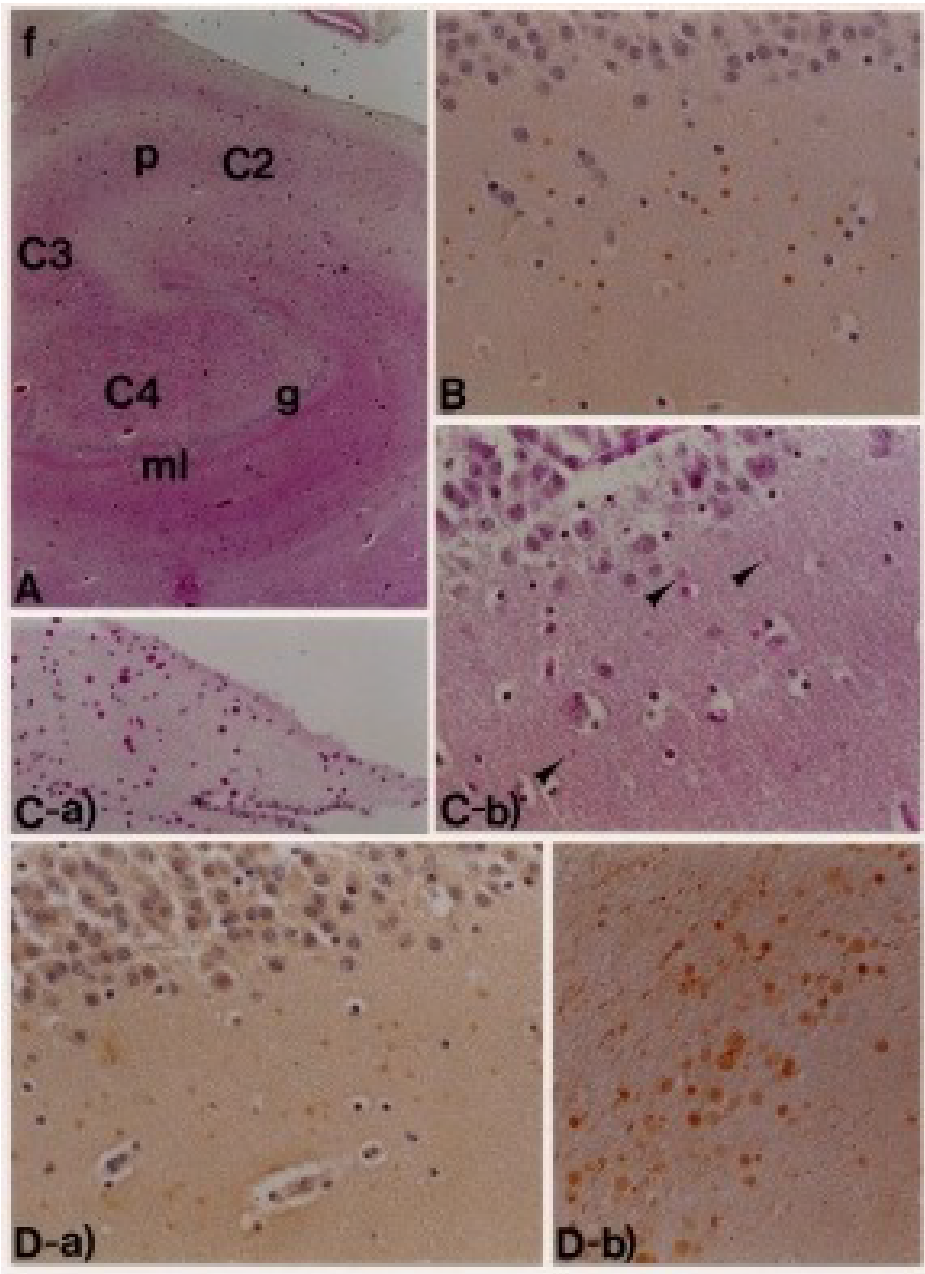

Figure: 1 (A): The hippocampal formation stained by Hematoxilin Eosin. g: granular layer p: pyramydal neuron ml: molecular layer f: fimbria (B): SPDs detected by lectin stains. GSI-B4. (C-a): Corpora amylacea are intensely stained by PAS. (C.b): SPDs are weakly stained by PAS. (D-a): SPDs show intense reactivity with antichondroitin sulfate. (D-b): Corpora amylacea show intense reactivity with anti-tau protein antibody.

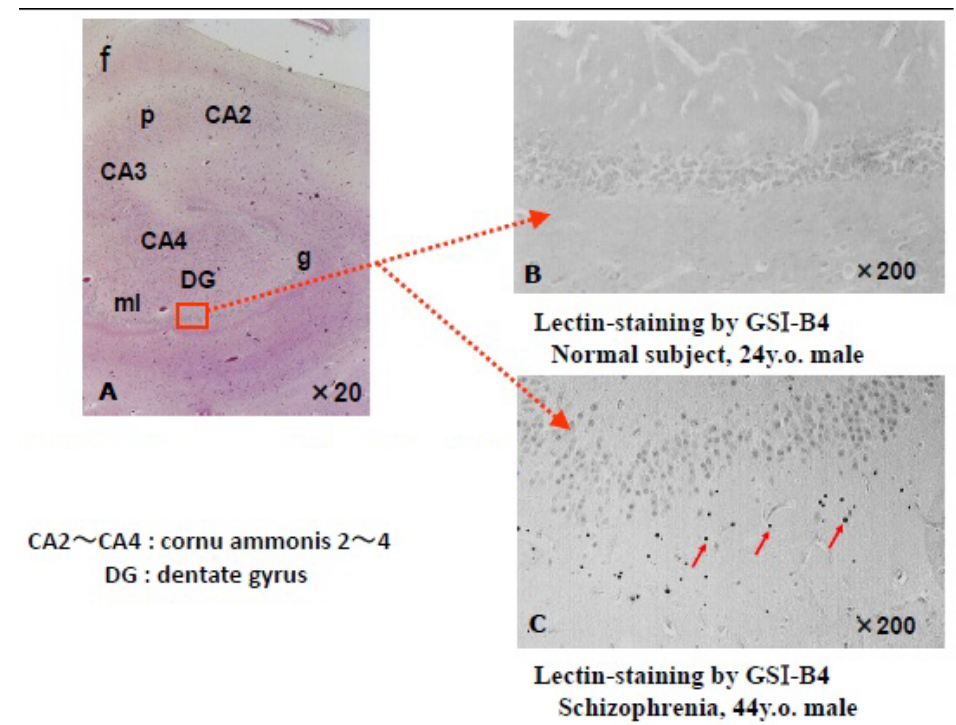

Figure: 2 The hippocampal formation (HE) (a) CA2 ${ }^{\sim}$ CA4: cornu ammonis $2 \sim 4$ DG: dentate gyrus (b) Lectin-staining by GS I -B4 Normal subject, 24y.o. male (c) Lectin-staining by GS I -B4 Schizophrenia, 44y.o. male 

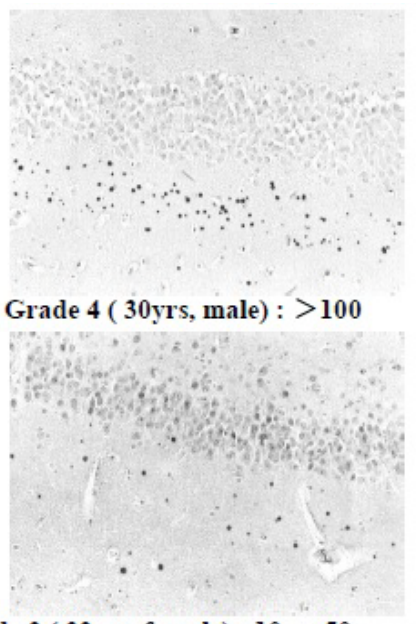

Grade 2 ( 32 yrs, female) : $10 \sim 50$
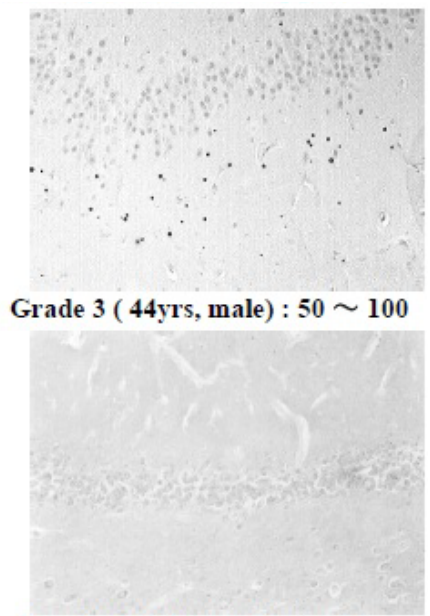

Grade 0 ( 24yrs, male) : none

Figure: 3 Frequency (Grade 1 4) of SPD appearance in molecular layer of hippocampal dentate gyrus (a). Grade 4 (30yrs, male): >100 (b). Grade 3 (44yrs, male): $50 \sim 100$ (c). Grade 2 (32yrs, female): $10 \sim 50$ (d) Grade 0 (24yrs, male): none

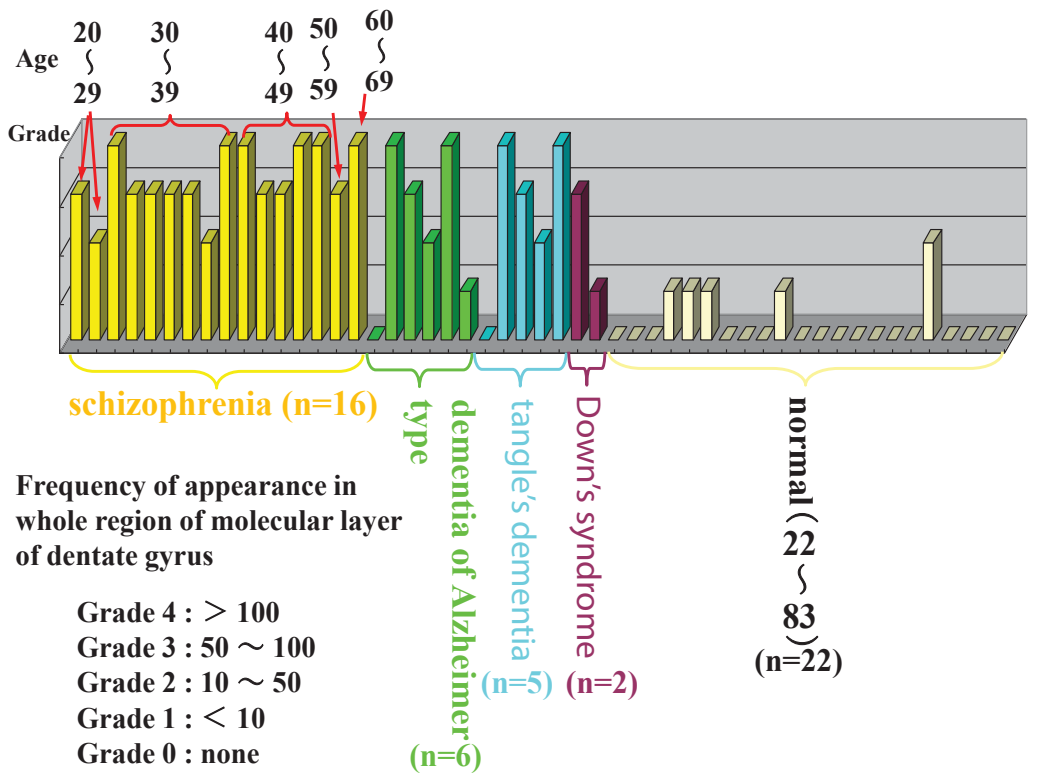

Figure 4: Frequency of lectin-positive SPD appearance.

Lectin-positive spherical deposits (SPD) in the molecular layer of hippocampal dentate gyrus

Lectin immunohistochemistry using various antibodies that detect sugar chains (Table 1), combined with conventional staining methods, revealed SPD, 3-10 micron in diameter, in the molecular layer of the dentate gyrus of the hippocampal formation (Figures 1-3) from patients with dementia of Alzheimer type, tangle's dementia, Down's syndrome and schizophrenia, and aged individuals (Figure 4) [2].

The lectin-positive SPD did not show immunoreactivity for antibodies against ubiquitin and tau-protein, though the corpora amylacea showed clear immunoreactivity for these substances (Figure. $1 D-b)[4]$. The SPD contained fucose ( $\alpha$-Fuc), galactose ( $\alpha$-Gal) (Figure 2C, 6), N-acetyl galactosamine (a-GalNAc) (Figure 5), N-acetyl glucosamine (GlcNAc), sialic acid, mannose (Man) and chondroitin sulfate (Table 1). Ultrastructural observation by electro-microscope showed that SPD morphology varied according to types of lectins (Figures 5-7).

\section{Lectin-positive SPD in schizophrenia}

In the brains of patients with schizophrenia, the lectin-positive SPD was observed in the molecular layer of the dentate gyrus of the hippocampal formation, without exception, regardless of previous medication of antipsychotics (Figure 8). In the lectin-positive SPD, partially disrupted nucleus with decreased staining properties by SYBR Green, a marker of DNA, were detected. Single strand DNA and lectin, including BSI-B4 for galactose (Figure. 7B-a, 7B-b), UEA-I for fucose (Figure. 7C-a, 7C-b), and DBA for N-acetylgalactosamine (Figure. 7Aa, 7A-b) were co-stained in the portion of partially disrupted nucleus (Figure. 7, Table 1). In immuno-electron microscope method, lectin- 


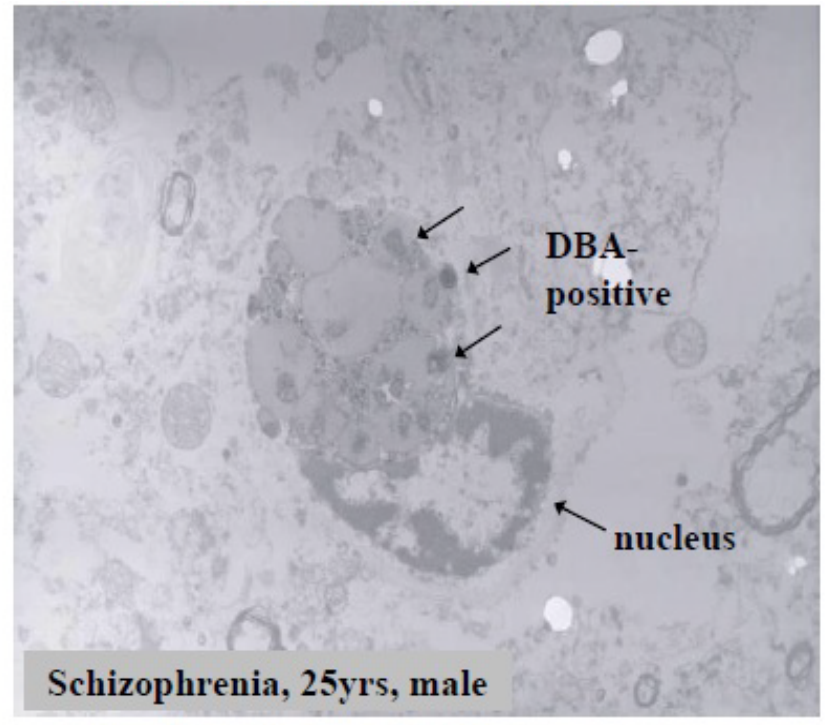

Figure: 5 Ultrastructure of a lectin-stained cell DBA-positive structures are shown by arrows. positive structures were detected in the portion of partially disrupted nucleus (Figure. 5, 6, 7A-b, 7B-b, 7C-b).

\section{Lectin-positive SPD in dementia}

In dementia of Alzheimer type, and tangle's dementia, some cases did not contain hippocampal lectin-positive SPD, being different from schizophrenia (Figure 4).

\section{Discussion}

Recent studies have shown more and more importance of the involvement of altered glycosylation in pathological procedures of neuropsychiatric diseases. In the superior temporal gyrus, altered $\mathrm{N}$-glycosylation of GABAA receptor has been shown in schizophrenia [7].

The SPD contained fucose ( $\alpha$-Fuc), galactose ( $\alpha$-Gal) (Figure $2 \mathrm{C}, 6$ ), $\mathrm{N}$-acetyl galactosamine ( $\alpha$-GalNAc) (Figure 5), N-acetyl glucosamine (GlcNAc), sialic acid, mannose (Man) and chondroitin sulfate, suggesting that there might be unusual glycometabolism (Table 1), possibly related to the process of neurogenesis in the molecular layer of hippocampal dentate gyrus (Figure 5-7). The lectin-positive structures, detected by electro-microscopic observation in the portion of partially disrupted nucleus (Figure. 5, 6, 7A-b, 7B-b, 7C-b) suggested that these
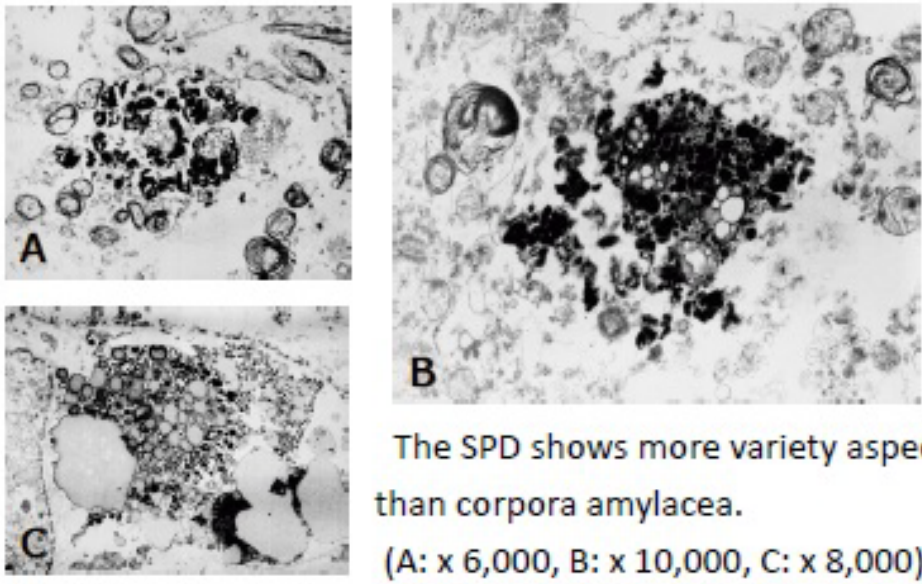

The SPD shows more variety aspect

than corpora amylacea.

(A: $x$ 6,000, B: x 10,000, C: x 8,000).

Figure: 6 Ultrastructure of SPD stained by GSI-B4. The SPD shows more variety aspect than corpora amylacea. (A: $\times 6,000$, B: $x$ 10,000, C: $\times 8,000)$.
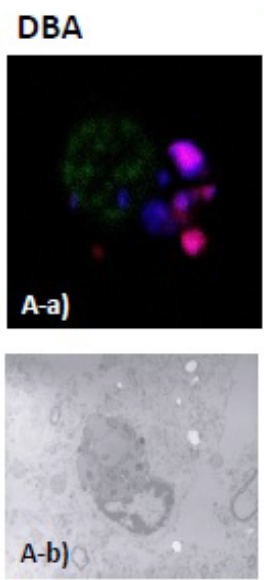

GSI-B4
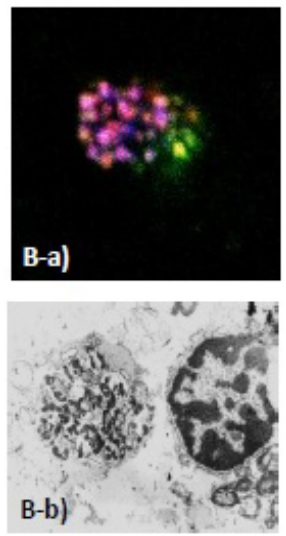

UEA-I
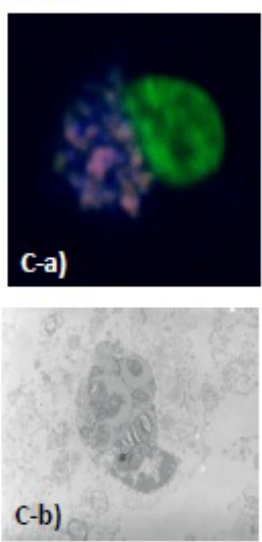

red: Lectin blue: Single strand DNA green: SYBR Green

Figure: 7 Lectin-positive SPD in hippocampus of schizophrenia (25 y.o. male) Immunofluorescence triple-staining and ultrastructural observation. 


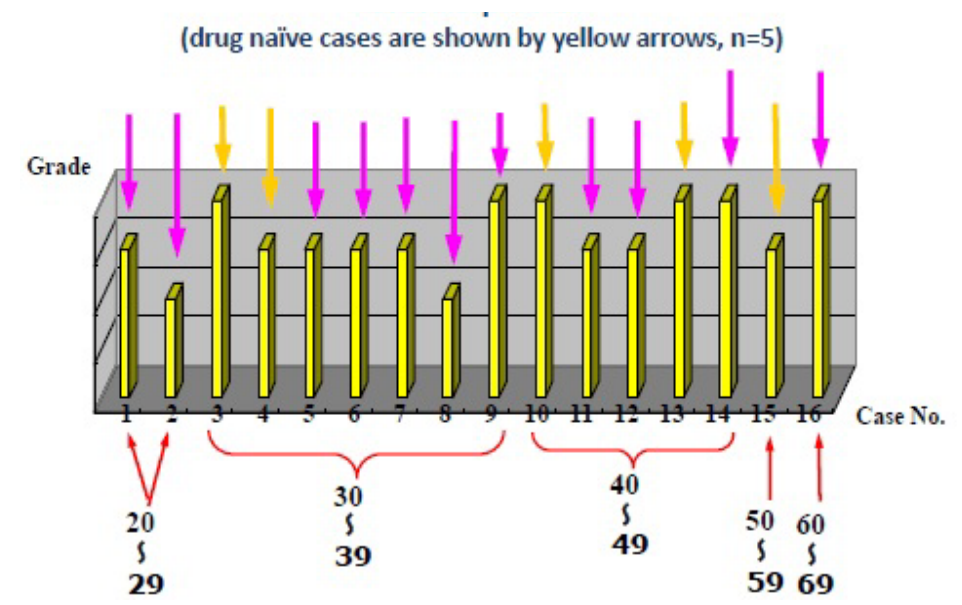

Figure: 8 Lectin-positive SPD was detected both in medicated and drug naïve cases of schizophrenia (drug naïve cases are shown by yellow arrows, n=5)

lectin-positive SPD might be produced in the process of apoptosis (Figure 7) [3,6]. The molecular basis of lectin-positive SPD formation of schizophrenia is shown to link with apoptotic process, and may also related to neurodevelopment.

In our study, the highlight is that the lectin-positive SPD in the molecular layer of dentate gyrus of hippocampal formation was observed in not only patients with clinical history of pharmacotherapy, but also in drug naïve patients, without exception. Though some experimental studies showed that a kind of therapeutic products of major tranquillizers induced neuron apoptosis in the dentate gyrus [8], the lectin-positive SPD in the hippocampal dentate gyrus may be schizophrenia specific.

Similar deposits, a phosphorylated tau-positive spherical inclusion, which increased with aging and decreased after occurrence of neurofibrillaty tangles, has been reported in the hippocampal CA1 region and the prosubiculum [9]. The size of the inclusion was reported to be 3-20 micron in diameter [9]. The lectin-positive SPD in hippocampal dentate gyrus shown in the present study was in a similar shape and diameter, 3-10 micron. The SPD did not show immunoreactivity for antibodies against tau-protein and ubiquitin, though the corpora amylacea showed clear immunoreactivity for these substances. The SPD was detected also in Tangle's dementia (Figure 4, 4 in 5 cases). Then, a part of lectin-positive SPD might possibly be overlapped with the phosphorylated tau-positive inclusion [9], however, molecular basis of both is likely to be distinct.

It should be detail analyzed the nature of lectin-positive SPD of schizophrenia as well as non-schizophrenia cases, including Parkinson's disease, dementia, Down's syndrome, and aged individuals (Figure 4) in relation between age- and/or disease-related deposits such as a phosphorylated tau-positive inclusion.

As novel therapeutic strategies, some pharmacodynamics studies have recently shown the usefulness of exogenous lectin for brain delivery of intranasally administered drugs of neuropsychiatric diseases such as schizophrenia [10] and Parkinson's disease [11].

It is important to elucidate lectin pathology as well as lectin physiology to establish novel methods for diagnoses and treatment of neuropsychiatric illnesses.

\section{Conclusion}

The molecular layer of hippocampal dentate gyrus of schizophrenia contained lectin-positive SPD, regardless of the history of pharmacotherapy. It was shown that hippocampal lectin-positive SPD formation in schizophrenia might be related to apoptotic process. To newly establish biomarkers of schizophrenia and other neuropsychiatric illnesses, the molecular basis of formation of lectin-positive SPD should further be analyzed.

\section{References}

1. Kolomeets NS, Uranova N (2010) Ultrastructural abnormalities of astrocytes in the hippocampus in schizophrenia and duration of illness: a postortem morphometric study. World J Biol Psychiatry 11: 282-292.

2. Kobata A (1998) A retrospective and prospective view of glycopathology Glycoconj J 15: 323-331.

3. Nishi $\mathrm{K}$, Tanegashima A, Yamamoto $\mathrm{Y}$, Ushiyama I, Ikemoto $\mathrm{K}$, et al. (2003) Utilization of lectin-histochemistry in forensic neuropathology: lectin staining provides useful information for postmortem diagnosis in forensic neuropathology. Leg Med (Tokyo) 5: 117-131.

4. Nishimura A, Ikemoto K Satoh K, Yamamoto $Y$, Rand S, et al. (2000) The carbohydrate deposits detected by histochemical methods in the molecular layer of the dentate gyrus in the hippocampal formation of patients with schizophrenia, Down's syndrome and dementia, and aged person. Glycoconj J 17: 815-822.

5. Ikemoto K, Amano R, Ishibe A, Nishimura A, Nishi K, et al. (2000) Quantitative analysis of tyrosine hydroxylase, aromatic L-amino acid decarboxylase or phenylethanolamine- $\mathrm{N}$-methyltransferase-immunoreactive neurons in the human medullary C1 region. Acta Histochemica et Cytochemica 33: 259-265

6. Nishimura A (2010) Are lectin positive spherical deposits detected in the molecular layer of the hippocampal formation related with neuronal apoptosis? J Med Invest 57: 183-190.

7. Mueller TM, Haroutunian V, Meador-Woodruff JH (2014) N-Glycosylation of GABAAreceptorsubunitsisalteredinSchizophrenia. Neuropsychopharmacology 39: 528-537.

8. Abekawa T, Ito K, Nakagawa S, Nakato Y, Koyama T (2011) Effects of aripiprazole and haloperidol on progression to schizophrenia-like behavioural abnormalities and apoptosis in rodents. Schizophr Res 125: 77-87.

9. Kimura T, Fujise N, Ono T, Shono M, Yuzuriha T, et al. (2002) Identification of an aging-related spherical inclusion in the human brain. Pathol Int 52: 636-642.

10. Piazza J, Hoare T, Molinaro L, Terpstra K, Bhandari J, et al. (2014) Haloperidolloaded intranasally administered lectin functionalized poly (ethylene glycol)block-poly(D,L)-lactic-co-glycolic acid (PEG-PLGA) nanoparticles for the treatment of schizophrenia. Eur J Pharm Biopharm. 87: 30-39.

11. Wen Z, Yan Z, Hu K, Pang Z, Cheng X, et al. (2011) Odorranalectin-conjugated nanoparticles: preparation, brain delivery and pharmacodynamics study on Parkinson's disease following intranasal administration. J Control Release 151: 131-138. 